\title{
Award Winners of JES
}

\section{JES Distinguished Service Award Winners}

\section{Winners in 2002}

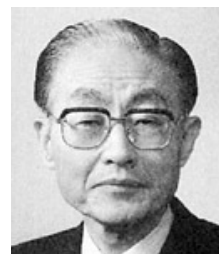

Kazuo Shizume, Honorary member Shizume Memorial Clinic

Present Affiliation: Shizume Memorial Clinic

Positions in JES:

Oct. $1973 \sim$ Jan. 1980

Jun. 5 7, 1976

Jan. $1980 \sim$ Feb. 1982

Feb. $1982 \sim$ May 1985

May $1985 \sim$ Mar. 1989

Jun. $1989 \sim$ Jun. 1991

$1994 \sim 2002$
Director

President of the $49^{\text {th }}$ Annual

Meeting of the JES

Chair, the Board of Directors

Chair, the Board of Directors

Auditor

Chair, the Board of Directors

Establishment of Shizume

Foundation

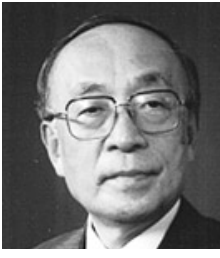

Hiroo Imura, Honorary member Council for Science and Technology Policy Present Affiliation: Foundation for Biomedical Research and Innovation

Positions in JES:

Oct. 1973 Jun. 1987 Director

Jun. 7 9, $1979 \quad$ President of the $52^{\text {nd }}$ Annual Meeting of the JES

Jun. $1987 \sim$ May 1989 Chair, the Board of Directors

Jul. 17 23, 1988

Jun. $1989 \sim$ Mar. 1991

Feb. $1993 \sim$ Jan. 1997 LOC Chairman, the $8^{\text {th }}$ ICE

Auditor

Jun. $1995 \sim$ May 1997
President of ISE

Chair, the Board of Directors

\section{JES Award Winners}

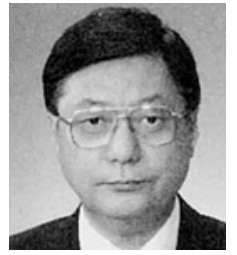

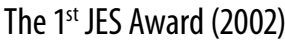

Hajime Nawata

Department of Medicine and Bioregulatory Science, Graduate School of Medical Sciences, Kyushu University "Basic and Clinical Research of Steroid Hormones and Their Receptors"

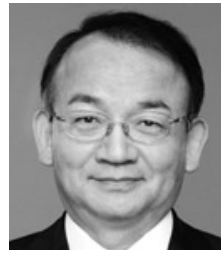

The $2^{\text {nd }}$ JES Award (2003)

Kazuwa Nakao

Department of Medicine and Clinical Science, Kyoto University Graduate School of Medicine "Research of Pathophysiological Significance and Clinical Application of Natriuretic Peptide Family and Leptin"

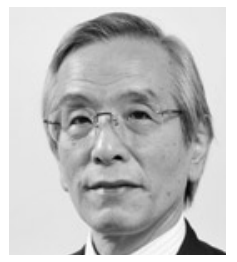

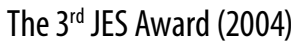

Kenji Kangawa

Department of Biochemistry, National Cardiovascular

Center Research Institute

"Discovery, Characterization, and Basic Research

of Novel Peptide Hormones"

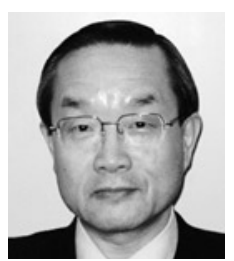

The $4^{\text {th }}$ JES Award (2005)

\section{Kazuo Chihara}

Division of Endocrinology/Metabolism, Neurology and Hematology/Oncology, Department of Clinical Molecular Medicine, Kobe University Graduate School of Medicine "Basic and Clinical Research of Growth Hormone"

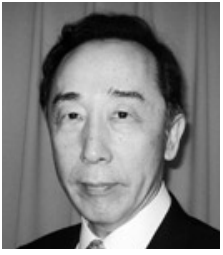

The $5^{\text {th }}$ JES Award (2006)

Masatomo Mori

Department of Medicine and Molecular Science, Gunma

University Graduate School of Medicine

"Research of Regulatory Factors of Thyroid Diseases and Obesity"

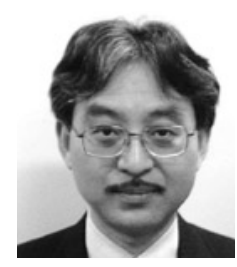

The $6^{\text {th }}$ JES Award (2007)

Shigeaki Kato

Institute of Molecular and Cellular Biosciences,

The University of Tokyo

"Research of Transcriptional Regulatory Functions of Nuclear Steroid Receptors"

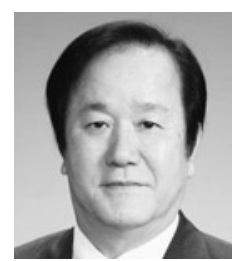

The $7^{\text {th }}$ JES Award (2008)

Toshio Matsumoto

Department of Medicine and Bioregulatory Sciences,

The University of Tokushima Graduate School of Health

Biosciences

"Research for Fusion Between Bone Calcium

Endocrinology and 0ther Research Fields"

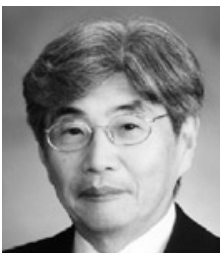

The $8^{\text {th }}$ JES Award (2009)

Masato Kasuga

Research Institute, International Medical Center of Japan

"Research of Insulin Actions and Pathogenic

Mechanisms of Diabetes Mellitus" 


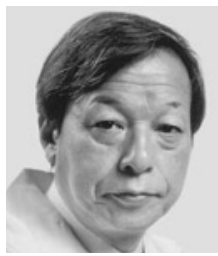

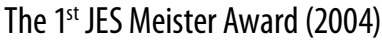

Shigetada Nakanishi

Department of Biological Sciences, Kyoto University

Graduate School of Medicine

"Molecular Mechanisms of Biogenesis and Reception

of Bioactive Peptides"

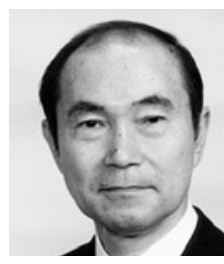

The $2^{\text {nd }}$ JES Meister Award (2005)

Katsuhiko Mikoshiba

Department of Basic Medical Sciences, Division of

Molecular Neurobiology, Institute of Medical Science, The University of Tokyo

"Research of Structure, Function, and Intracellular

$\mathrm{Ca}^{2+}$ Regulation of $\mathrm{IP}_{3}$ Receptor"

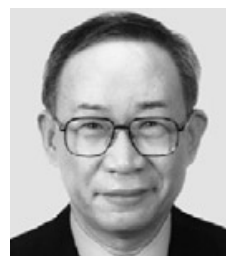

The $3^{\text {rd }}$ JES Meister Award (2006)

Shuh Narumiya

Department of Cell Pharmacology, Kyoto University

Graduate School of Medicine

"Molecular Cloning, Signal Transduction,

Physiological Functions, and Pathogenic Significance

of Prostaglandin Receptors"

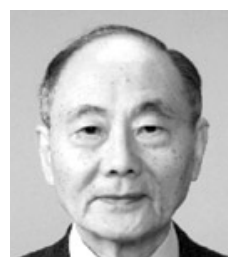

The $6^{\text {th }}$ JES Meister Award (2009)

Kiyoshi Miyai

Professor Emeritus of Osaka University

"Research of Congenital Pituitary and Thyroid

Disorders"

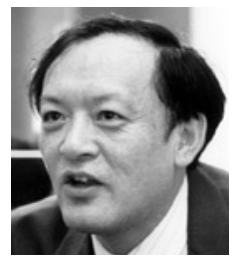

The $7^{\text {th }}$ JES Meister Award (2010)

Shigekazu Nagata

Department of Medical Chemistry, Kyoto University

Graduate School of Medicine

"Engulfment and Degradation of Apoptotic Cells"

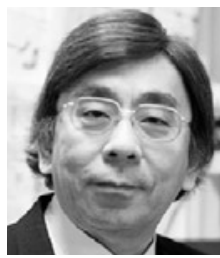

The $4^{\text {th }}$ JES Meister Award (2007)

Nobutaka Hirokawa

Department of Cell Biology and Anatomy, Graduate

School of Medicine, The University of Tokyo

"Intracellular Transport and Kinesin Superfamily

Motor Proteins, KIFs: Structure, Function, and

Dynamics"

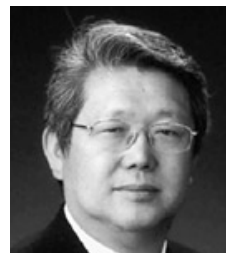

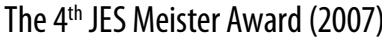

Yuji Matsuzawa

Sumitomo Hospital, Professor Emeritus of Osaka

University

"Research of Adipocytokines Secreted From

Adipocytes" 


\section{JES Best Endocrine Surgeon of the Year Winners}

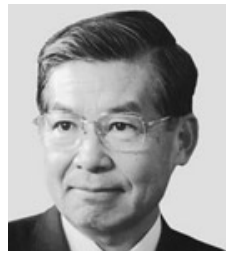

The $1^{\text {st }}$ JES Best Endocrine Surgeon of the Year (2004)

Masayuki Imamura

Professor Emeritus of Kyoto University

"Surgical Treatment of Pancreatic Endocrine Tumors"

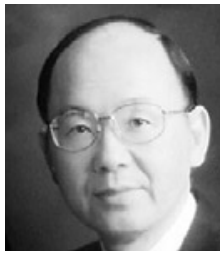

The $4^{\text {th }}$ JES Best Endocrine Surgeon of the Year (2007)

Masaru Murai

International Goodwill Hospital, Professor Emeritus of

Keio University

"Laparoscopic Adrenalectomy"

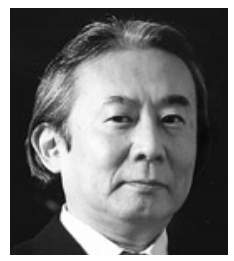

The $2^{\text {nd }}$ JES Best Endocrine Surgeon of the Year (2005)

Akira Teramoto

Department of Neurosurgery, Nippon Medical School

"Development of Diagnosis and Surgical Treatment of

Pituitary Tumors"

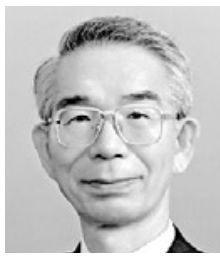

The $5^{\text {th }}$ JES Best Endocrine Surgeon of the Year (2008) Akira Miyauchi

Kuma Hospital

"Elucidation of Pathogenic Mechanism of Acute Pyogenic Thyroiditis, Discovery of ITET/CASTLE, and Innovation of Recurrent Laryngeal Nerve Reconstruction"

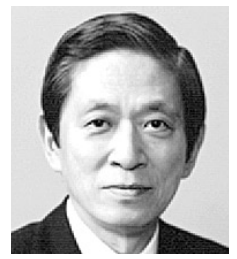

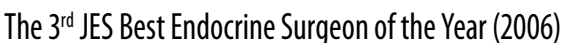

Hiroshi Takami

Department of Surgery, Teikyo University

"Advances in Surgical Treatment of Thyroid and

Parathyroid"

\section{JES Distinguished Endocrinologist Award Winners}

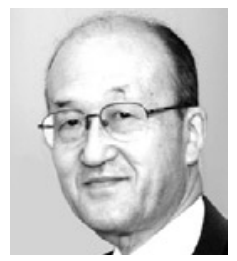

The $1^{\text {st }}$ JES Distinguished Endocrinologist Award (2009)

Yoshiyuki Osamura

Division of Diagnostic Pathology, Tokai University School of Medicine

"My Pituitary Research—Pathology and Endocrinology"

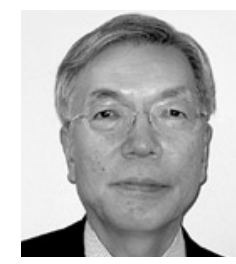

The $2^{\text {nd }}$ JES Distinguished Endocrinologist Award (2010)

Tetsuo Nishikawa

Yokohama Rosai Hospital

"Problem Solving in Diagnosis and Treatment of

Primary Aldosteronism" 